\title{
COLLAPSE THERAPY (MEDICAL) IN PULMONARY TUBERCULOSIS
}

\author{
By F. H. Scadding, M.D., M.R.C.P. \\ Assistant Physician, Middlesex Hospital
}

Whatever the exact mechanism involved in the various measures embraced by the term ' collapse therapy' for pulmonary tuberculosis, a feature common to them all is the reduction in volume of the diseased portion of lung. There is ample evidence that the resultant relaxation is beneficial and conducive to healing. Yet, even before the advent of chemotherapy and the progress in thoracic surgery which the past decade has witnessed, the mainstay of minor collapse therapy, the pneumothorax, was receiving its full share of criticism. Unfavourable general conclusions, however, were too often drawn from what we should now regard as its misuse. Many past failures can today be understood as a result of our greater knowledge of the pathology of the disease, especially as regards the important role played by the bronchi. The essential factors for success of any treatment are a proper selection of cases and a clear recognition of the limitations of the particular method employed. All are agreed that cavitated disease is the main indication for some form of collapse therapy. In such cases the risk of further spread of the disease by bronchial aspiration to the opposite lung or to other parts of the same lung is ever present, and the ultimate prognosis of patients with persistent cavitation is very poor indeed. Bed rest alone, if strict and prolonged, will cause some cavities to close. If, at the same time, a position in bed is adopted which places the cavity in the most dependent position, as suggested by Dilwyn Thomas (r950), even large cavities can sometimes be persuaded to close or shrink considerably in size. Without additional mechanical control, however, the tendency for them to re-open when the patient becomes ambulant is very great. Effective antibacterial treatment of recent years raised hopes that collapse procedures might soon become unnecessary. Such hopes, however, have not yet been realized. Recent pulmonary cavities will often appear to close on chemotherapy, but if no additional measures are adopted, the relapse rate is high when the treatment is stopped. For disease which has progressed to cavitation, there-o fore, chemotherapy must be integrated with othez forms of treatment and not replace them. It has 3 . done much to make them safer by rendering thew disease quiescent before collapse therapy, and $\omega$ has also reduced the incidence of the more serious + complications; to a lesser extent it has broughtio unsuitable cases within reach of collapse therapy.A

Whether to apply collapse measures for un-음 cavitated tuberculous infiltration remains a difficult problem, the solution of which depends $\_$ largely on personal judgment and experience. Assuming there is evidence of activity, anti- $\overrightarrow{0}$ bacterial treatment should be started withopit delay together with bed rest, preferably under close observation in hospital. If consideralieo radiological clearing does not result after six s weeks of such a regime, or should there beo deterioration, some form of collapse should beo considered. Many factors other than the X-ray appearance and clinical condition weigh in this decision, e.g. the young woman with dependent family, a bad family history of tuberculosis, raciae predisposition-such features would rightly in? fluence the decision for active treatment in caseso of doubt.

\section{Phrenic Interruption}

Various operations on the phrenic nerve from? simple freezing with ethyl chloride to avulsion were frequently practised in the r930s, but todays the only one in regular use is phrenic crush. It produces only a very moderate degree of pul monary relaxation and, although not quites obsolete, as a sole measure of collapse therapy it has a very limited sphere of application. To have any measurable effect the diaphragm should bew mobile prior to the operation, and the lung should not be widely adherent to the chest wall. Pres? liminary fluoroscopy will give some idea of the expected rise of the diaphragm, which on the average will be 2 to $4 \mathrm{~cm}$. The effect of a single crush usually lasts from six to nine months, but it should be borne in mind that about $5^{\circ}$ per cent $\frac{?}{\Phi}$ 
obtain only a partial recovery of function, and more than io per cent. do not recover at all. Regeneration proceeds more slowly with increasing age, so this operation should seldom be used in patients over fifty. It is quite useless for extensive fibrous disease or thick walled cavities, and should be avoided in patients likely to require major surgery in view of the higher incidence of postoperative complications. It still has advocates for its trial in patients with limited exudative disease whose response to rest and chemotherapy is slow. Its main role, however, is a supplementary one, either as an adjunct to a pneumopsritoneum, or to improve an inadequate pneumothorax in which, because of apical and basal adhesions, the affected lung is only relaxed in the lateral plane. At operations for lung resection it can be used to diminish the capacity of the hemithorax and, at the termination of a pneumothorax, particularly if the lung is slow to re-expand, a phrenic crush will enable a more gradual adjustment of the expanding lung and help to lessen any tendency to mediastinal displacement. If used for this purpose the operation should be done before the lower lobe makes contact with the chest wall.

\section{Artificial Pneumothorax}

There are several reasons for the waning popularity of pneumothorax treatment of pulmonary tuberculosis over the past ten years. General conclusions drawn from its use in the wrong types of case have in part been responsible. Other important factors are the introduction of a simple and safe alternative in the form of pneumoperitoneum, the availability of effective chemotherapy and the great strides made by thoracic surgery. Nevertheless, a good pneumothorax is still the most effective minor collapse procedure, since it provides concentric relaxation and is applicable to disease in any part of the lung. It must be realized, however, that many forms of pulmonary tuberculosis are unsuitable for A.P. treatment, either because it is ineffective or positively dangerous. Experience has taught us to recognize the following contraindications:

(i) Tuberculous Pneumonias. Included in this group are also the more florid forms of the disease with extensive infiltration and clinical evidence of severe toxaemia. In such cases chemotherapy, possibly assisted by a pneumoperitoneum, should be the first line of treatment and may in some instances render subsequent A.P. treatment safe, but the induction of a pneumothorax in the early stages carries a great risk of pleural effusion and empyema.

(ii) Tuberculoma. The solid round focus may represent a blocked cavity, a circumscribed area of caseous pneumonia or a cold abscess of a bronchus. An A.P. has no material effect on such lesions and should therefore not be used.

(iii) Chronic Disease with Extensive Fibrosis or much Destruction of Lung Tissue. An effective A.P. is seldom obtainable because of indivisible pleural adhesions and, in general, such cases demand a more permanent form of relaxation, or resection.

(iv) Large Peripheral Cavities. These are almost always adherent to the chest wall and may receive part of their blood supply through such adhesions. If these should be severed the wall may necrose and allow the cavity to rupture into the pleural space with disastrous consequences.

(v) Tuberculous Disease of Major Bronchi. Tuberculous bronchitis should be suspected where the X-ray shows evidence of atelectasis or a 'tension' type of cavity. The narrowing of a bronchus caused by tuberculous inflammation of its wall may result in a check-valve mechanism whereby air may enter a cavity under conditions of raised intra-bronchial pressure, as during coughing, but be unable to escape during expiration. Such a cavity tends to be spherical, thinwalled and often contains a fluid level in view of the impaired bronchial drainage. Permanent closure of the affected bronchus will result in cavity closure, and this can sometimes be achieved by an A.P. Unfortunately, however, the checkvalve effect may only be aggravated with a consequent rapid increase in size of the cavity and possible rupture. It is wiser, therefore, to avoid a pneumothorax in such cases unless the tension element in the cavity can first be overcome by chemotherapy.

The exclusion of all the above groups decidedly narrows the field for A.P. treatment in pulmonary tuberculosis. Nevertheless, there remains a considerable number of patients with recent scattered infiltration, often with cavities of less than $4 \mathrm{~cm}$. diameter, and mainly or entirely unilateral distribution. For these a pneumothorax is most useful. Its induction, however, should be regarded as an exploratory procedure and it should be abandoned if it is not effective or cannot be made effective by adhesion section. If feasible, all adhesions preventing relaxation of the diseased area should be divided within the first few weeks of induction. Considerable experience is required to decide on the safety and extent of this operation, as the most serious complications of A.P. treatment often date from it.

The importance of undivided adhesions is still a matter of controversy. There is, however, general agreement that a contra-selective pneumothorax, or one under which a cavity persists, should not be maintained. They are fraught with danger and, as Rafferty (1947) has shown, the 
prognosis for such patients is worse than if they had no active treatment. Some authorities consider that only those A.P.s should be maintained in which the lung is completely free. A more moderate view was expressed by Scadding et al. (I95I), who found that adherence of the upper lobe to the superior mediastinum did not necessarily preclude success nor carry any great risk. Foster-Carter et al. (1952) reviewed 457 patients treated by artificial pneumothorax at Brompton and its sanatorium five years after their A.P.s had been given up. The survival rate in patients with a completely free lung was $9 \mathrm{I}$ per cent. and, in the 'adherent satisfactory' group of 242 patients, where adhesions to the diseased area were present but did not prevent cavity closure within a year, the survival rate was 94 per cent. Furthermore, the incidence of persistent pleural effusions was the same in the two groups. They concluded that the key to successful pneumothorax treatment was closure of cavities and not the presence of indivisible adhesions. With proper selection most cavities will close within two months, but a few may take several months. In the absence of complications, therefore, an imperfect pneumothorax need not be abandoned in favour of surgery before its merits have been fairly assessed after a trial period of adequate and frequent refills.

\section{Pleural Effusion and 'Frozen' Lung}

A small collection of fluid in the costo-phrenic angle is frequently observed during treatment by pneumothorax. Remaining below diaphragm level, it seldom interferes with the course of the A.P. and requires no treatment other than observation. Occasionally it appears to initiate premature obliteration of the pleural space, but is otherwise harmless. The larger, cumulative effusions, however, are of more serious import. If they do not absorb within a few weeks of treatment by extra rest, aspirations and lavage, it is probably wise to abandon the A.P., even though more drastic surgical treatment may then have to be faced. Evidence is accumulating to show that initial chemotherapy reduces the incidence of these effusions and of tuberculous empyema following adhesion section.

The factors which predispose to the complication of 'frozen' lung are the persistent pleural effusion, extensive pulmonary fibrosis and permanent atelectasis. It can often be anticipated and, therefore, should be largely preventable by avoiding A.P. treatment in cases with bronchial disease or a considerable fibrotic element, and by the early abandonment of those pneumothoraces in which progressive pleural thickening or effusion occurs. Once the condition is fully developed the loss of pulmonary function can seldom be regained even by decortication of the affecteक्ष lung (Wright et al., 1949).

\section{Duration and Termination}

The aim of treatment is to continue the pneumo thorax until the disease is healed, but to give it up while the lung is still capable of re-expansiono There can be no fixed rule about its duration since such variable factors as the character an extent of the original lesion, age and sex of the patient, type of pneumothorax and its complicas tions have to be considered in each case. In general, a period of three years will be required? with extension to five years if cavities were present initially. It may well be that chemotherapy in the early months of treatment may shorten the time necessary for healing, but there is not ye sufficient evidence to warrant any drastic reduction in the period of collapse. When the decision t\& discontinue the A.P. has been taken, a progressivt reduction in either the frequency or the amounity of refills is preferable to an abrupt cessation. The more gradual adjustment minimises the patient' $\$$ discomfort and diminishes the risk of late effusiom or tearing of an indurated visceral pleura. Tomo graphy should be done before the lung reaches and adheres to the chest wall as, in the few whose cavities reopen, the pneumothorax may be regaige if further minor collapse therapy is thought likely to succeed.

\section{Pneumoperitoneum}

The introduction of air into the peritonea cavity as a treatment for bilateral pulmonare tuberculosis was suggested by Vajda in 1933 Banyai (r934), combining it with phrenic paralysiş explored its possibilities as a definitive form of collapse therapy, with favourable results, and since 1943 it has been widely practised in this country. It came as a welcome alternative to the pneumothorax and, indeed, it has certain advan tages over it in that the technique is easy to acquire serious complications are rare, it can be given up and re-induced at will, it can safely be used in the patient's own home and, above all, it is free from serious pleural and pulmonary complications Furthermore, it can be applied to the acute forms. of the disease, bilateral cases, and those in whicf a satisfactory A.P. cannot be obtained. In some chest clinics it has almost replaced artifici pneumothorax as the first choice when collapse therapy is indicated, and a large volume of medical literature testifies to its value. Most of the enthusiastic reports, however, are concerned onl\$ with early results.

The effect of pneumoperitoneum, generall speaking, is proportional to the degree of diaphrago matic elevation obtained, and little benefit can bo 


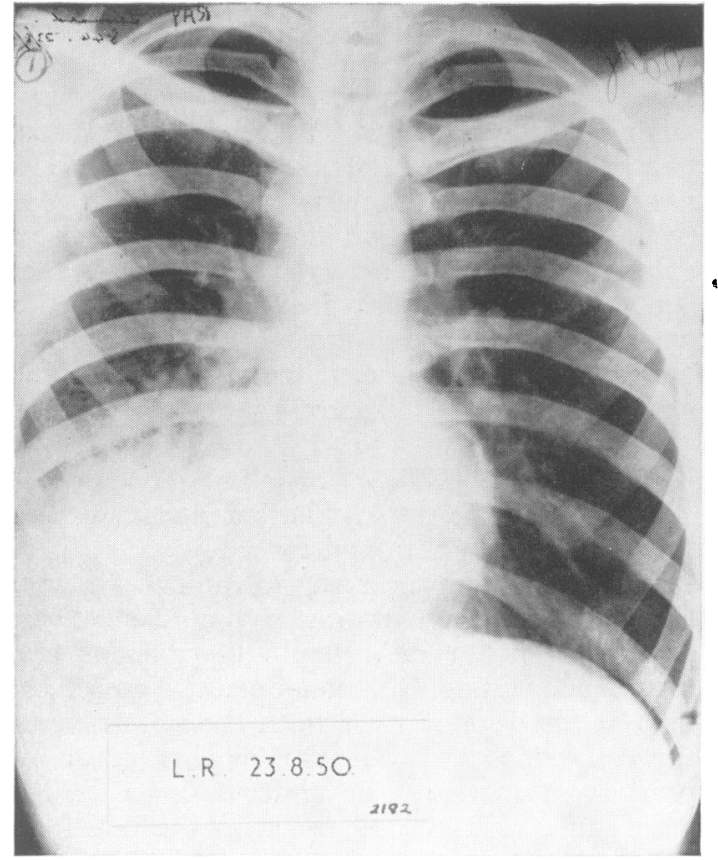

FIG. I (a).-Tuberculous pneumonia. Adolescent male, acutely ill with high fever and severe toxaemia; signs of consolidation right lower lobe and infiltration left mid-zone. Sputum positive.

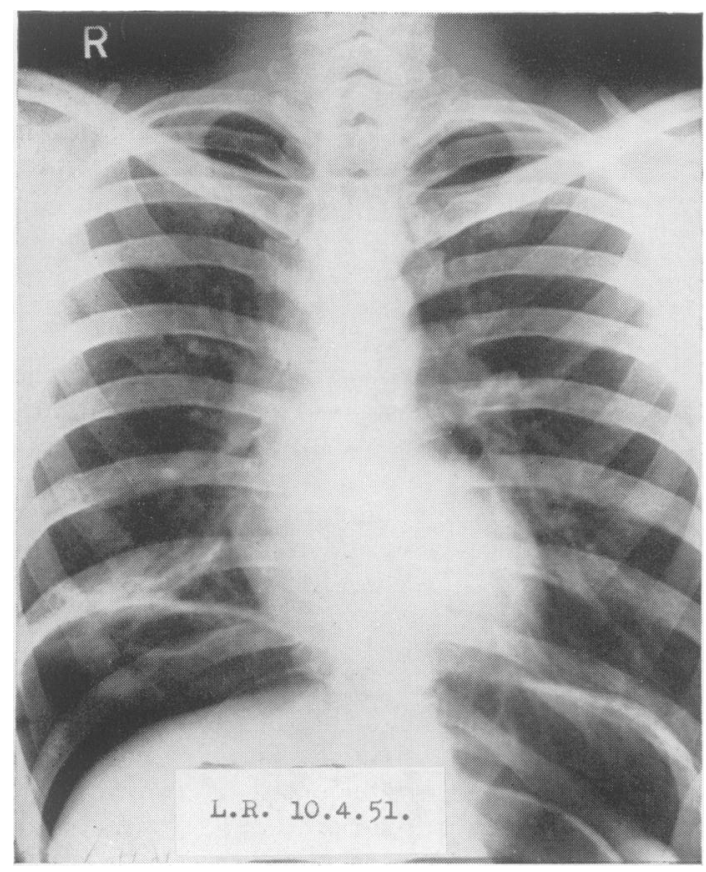

Fig. I (c).-After seven months chemotherapy combined with right phrenic and P.P. The diaphragm has recovered. Patient symptom-free, having gained over a stone in weight and beginning to get up. One gastric lavage culture positive.

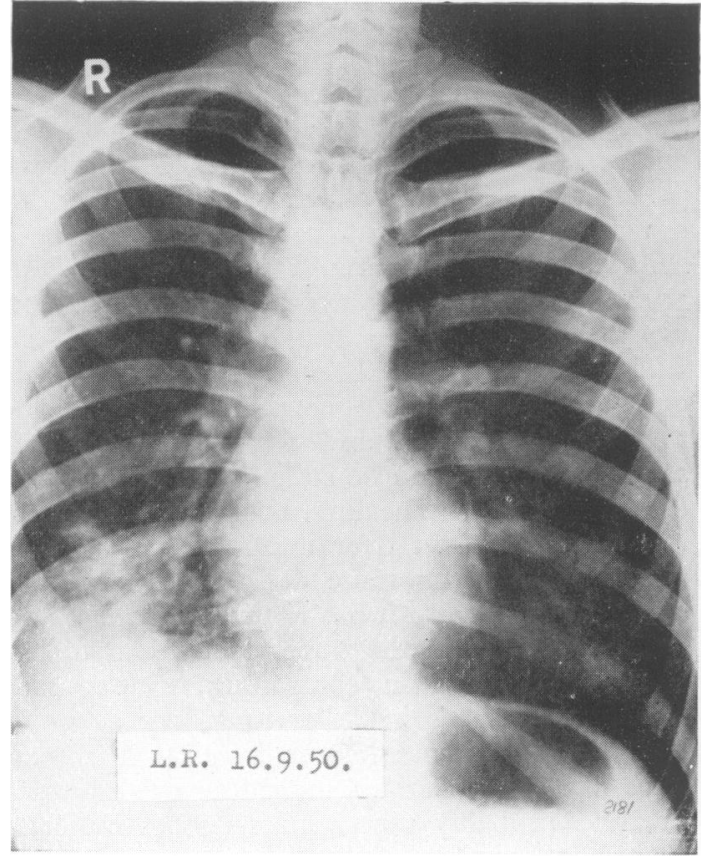

FIG. I (b).-After three weeks treatment with daily streptomycin and P.A.S. Some clinical improvement but lesion rapidly breaking down to form several cavities. Right phrenic crush and P.P. instituted.

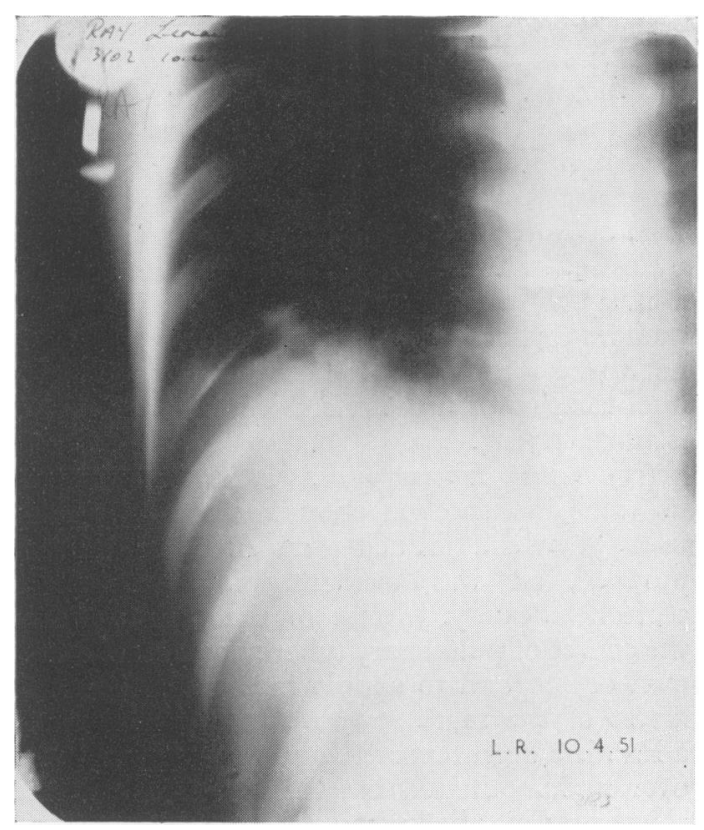

FIG. I $(d)$.-Tomogram showing persistent cavitation in right lower lobe. Two shrunken, bronchiectatic segments subsequently resected from right lower lobe without incident. Patient well and working to date. 
expected if the diaphragm is fixed, or if the pleura is widely adherent. It is quite common for the paralyzed diaphragm to rise to the level of the third rib anteriorly or even higher. Under such circumstances it can control lesions in the upper parts of the lung as effectively as those in the lower, provided they are of a type likely to respond (Anderson and Winn, 1945; Keers, 1948). The following types of disease respond well:

(i) Acute Exudative Lesions. In cases of tuberculous pneumonia the combination of chemotherapy with a P.P. and phrenic is often lifesaving. It may even be sufficient to bring quiescence and eventual healing, but more often serves to tide the patient over a critical phase of his illness and enables more definitive treatment at a later date. Whilst it has virtually no effect upon chronic cavities in the upper lobes, it may offer considerable help in controlling acute spreads from such cavities in either lung, and so render the patient acceptable for surgical treatment. This use of pneumoperitoneum as an interim measure probably represents its most important sphere of application in the management of pulmonary tuberculosis.

A good example is seen in Figs. I $(a)$ to $(d)$.

(ii) Predominantly Exudative Disease with Recent Cavitation, in which an A.P. has Failed or is Contraindicated. In such conditions a P.P. may be used with safety and is certainly preferable to maintaining an inadequate pneumothorax.

(iii) Lower Lobe Disease. A common site for pulmonary tuberculosis is the apical segment of the lower lobe, and in this situation it has often progressed to cavitation by the time it is diagnosed. Crofton (1949) showed that a good pneumothorax would close two-thirds of such cavities, and this is probably the treatment of choice, provided the draining bronchus can be shown at bronchoscopy not to be stenosed. If, however, a good A.P. is unobtainable then the effect of a P.P. and phrenic is worth trying.

(iv) Severe Haemoptysis. It is of interest that its value in this connection was noted by Banyai in 1931 when, inducing an A.P. for the same purpose, air was accidently injected into the peritoneal cavity. Most of the haemoptyses in the course of pulmonary tuberculosis cease on their own or respond to simple measures. Successful arrest of the more severe cases by induction of a P.P. has been reported, and it has the advantage over pneumothorax that the site of the haemorrhage need not be known.

\section{Complications}

Although the safety of the procedure has been stressed, more than 40 complications have been described, varying in severity from vague indigestion to sudden death. Perforation of abdominal viscera has often been reported but causes surprisingly little anxiety. The more serious complications include air embolism, intraperitoneal haemorrhage, spontaneous pneumothorax, mediastinal emphysema, torsion of the spleen and tuberculous peritonitis. Luckily they are all uncommon. Congestive heart failure may be precipitated in patients with a poor cardiac reserve, and an increased incidence of acute appendicitis has been noted (Rilance and Warring, 1944).

\section{Duration of Treatment}

Where a P.P. is employed as an interim measure it will usually be required for a few months, but the time will vary according to the circumstances of each case. The optimum time for surgery, and whether the P.P. should be abandoned or $\dot{\omega}$ maintained through the post-operative period, are $\vec{A}$ matters for discussion between the physician and is surgeon concerned. If the diaphragm is still $\stackrel{\oplus}{\perp}$ paralyzed some surgeons prefer to keep the P.P. 윽 going until after the operation in the belief that the risk of post-operative atelectasis is thereby $c$ lessened.

As with pneumothorax, there can be no set $\overrightarrow{0}$ time for which a P.P. should be maintained whed of it is the only means of collapse adopted. It unlikely to prove effective in a shorter time tha⿳亠. would a pneumothorax and a minimum period of three years is, therefore, advised. During this time it may be necessary to re-crush the phrenic nerve on more than one occasion, but it is often $\stackrel{\square}{\square}$ observed that the diaphragm remains high in $\overrightarrow{\vec{O}}$ position even after recovering its function. At 3 the termination of the treatment no ill effects $\underset{F}{\vec{F}}$ result from the abrupt cessation of refills, and the air has usually absorbed in 6 to 12 weeks.

In the absence of a specific remedy it takes many years to assess the value of any treatment for a $\frac{0}{3}$ disease with such a variable natural course as. pulmonary tuberculosis. The simplicity and relative harmlessness of pneumoperitoneum treat- 3 ment have encouraged its trial in all varieties of음 the disease, but the temporary relief of symptoms $D$ and psychological benefit sometimes afforded do을. not justify its continued use in unsuitable cases. N The late results are only just appearing and they are disappointing in advanced cases (Morris et al., $\mathbb{N}$ 1952). Edge (1953) confirms this, and reports also a high relapse rate (9 out of 25) soon aftero abandonment in patients with anything more thano early lesions. It seems likely that such results, together with further progress in other forms of ${ }^{\text {? }}$ treatment, will cause pneumoperitoneum to be $\frac{T}{0}$ used with greater discrimination.

Continued on page 304 
length of the fourth rib is resected. The extrapleural plane is entered through the rib bed and the apex of the lung is stripped off the endothoracic fascia to a similar extent to that for the two preceding operations. When absolute haemostasis has been achieved, and this may be difficult, the wound is closed with particular care to ensure that the intercostal layer is air tight. The collapse is then maintained by filling the space with air at suitable intervals.

\section{Results of Collapse Operation}

As the extent and the type of tuberculous lesions which are subjected to surgery vary so enormously, the statistical results in any series must depend to a very large degree on the type of case operated upon. For instance, if a high proportion of the patients are suffering from advanced bilateral disease the mortality rate may well be as high as Io per cent., whereas if the majority of the operations performed were for ' minimal' apical lesions without cavitation, no, mortality greater than I per cent. would be acceptable.

The overall mortality for thoracoplasty is approximately 3 per cent., and it is the same for plombage operations. In published series (Price Thomas, 1952; Sellors, 1947; Laird, 1953) it is in the same order.

In terms of sputum conversion the results of most collapse operations give figures ranging around 80 per cent. successes, but before conversion can be accepted at least three negative sputum or gastric lavage cultures are required, as the more thorough the search for bacilli the more often they are found. Short of post-mortem evidence cavity closure is almost impossible to prove. In terms of return to their previous occupation Price Thomas states that in his experience over 80 per cent. of the patients surviving operation are back in their original job. Early post-operative morbidity is higher after thoracoplasty than after plombage operations, but late tuberculous infection of the extra-periosteal space is the bugbear of the latter operation in which foreign bodies are left in situ, and it still occurs sufficiently often for many surgeons to doubt the justification of the pro- cedure. This criticism also applies to extra-0 pleural pneumothorax, in which late tuberculous infection is not uncommon; this operation has the 3 added disadvantage that refills are required weekly ${ }_{\infty}^{\mathbb{Q}}$ and that despite this inconvenience the lung.. seldom re-expands when refills are discontinued $\Rightarrow$ and so its basic purpose is not achieved.

\section{Summary}

I. Surgical collapse therapy has a large place in $\frac{\text { के }}{\widehat{D}}$ the treatment of pulmonary tuberculosis.

2. The decision as to the extent and the type of collapse should be based on as complete as possible $\vec{\circ}$ anatomical and pathological diagnosis of the disease. $-\overrightarrow{-}$

3. Thoracoplasty has the disadvantage that it $\vec{\omega}$ has to be done in more than one stage, but the long-term results are probably better than those in any other collapse operation.

4. Extra periosteal plombage has the advantage of being a one-stage procedure, but the dis- $\omega$ advantages inherent in any operation in which foreign material is left in situ.

5. Extra-pleural pneumothorax has a high morbidity and seldom achieves its object, that is that it ${ }^{\circ}$ can be abandoned when the underlying disease is ${ }_{\subset}$ controlled.

\section{BIBLIOGRAPHY}

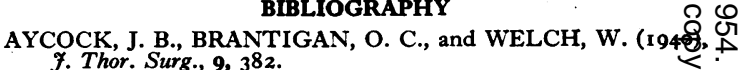

BAER, G. (1913), Munsch. Med. Wschr., 40, 1587.

EDWARDS, F. R. (1949), Thorax, 4, 224.

GRAF, W. (1936), Dtsch. Med. Wschr., 62, 632.

GRENVILLE-MATHERS, R., and TRENCHARD, W. J. (1952), $\bar{O}$ Thorax, 7,185 .

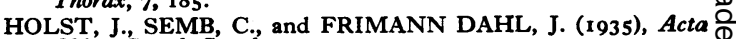
Chirg. Scand. Supplement 37.

LAIRD, R. (1953), Lancet, 265, 319.

LUCAS, B. G. B., and CLELAND, W. P. (1948), Thorax, r, 2 r1. MORRISTON DAVIS, $W$., and TEMPLE, J., and STATHATOS, 3 C. (195I), Ibid., 6, 209.

MORRISTON DAVIS, W. (r933), 'Pulmonary Tuberculosis Medical and Surgical Treatment,' London, Cassell.

PRICE THOMAS, C., and CLELAND, W. P. (1942), Brit. $\mathscr{Y} \cdot \frac{\partial}{\partial}$ Tub., 36, rog.

PRICE THOMAS, C. (1952), 'Modern Practice in Tuberculosis, Sellors, Livingstone \& Butterworth.

SAUERBRUCH, F. (1920), 'Die Chirurgie de Brustorgane.'

SEMB, C. (1935), Acta Chirg. Scant. Supplement 37

SELLORS, T. H. (1948), Thorax, 2, 216.

SELLORS, T. H. (1953), personal communication

TUFFIER, T. (1895), Gaz Hosp. Paris, 68, 1320.

WILSON, D. A. (1946), Surg. Clin. N. Amer., 26, 1060.

Continued from page 298-P. H. Scadding, M.D., M.R.C.P.

\section{Acknowledgments}

I am grateful to Mr. D. F. Kemp, of the photographic department of the Institute of the Diseases of the Chest, for the X-ray photographs in Figs. I $(a)$ to $(d)$.

\section{BIBLIOGRAPHY}

ANDERSON, N. L., and WINN, W. D. (1945), Am. Rev. Tub.,

BANYAI, A. L. (1946), 'Pneumoperitoneum Treatment,' Henry Kimpton, London.
CROFTON, J. W. (1949), Thorax, 4, 96.

EDGE, J. R. (1953), Brit. F. Tuberc., 47, 202 . YOUNG, F. H., and BENJAMIN, B. (1952), Brompton Hospital Reports, Vol. xxii.

KEERS, R. Y. (1948), Brit. $\mathcal{Y}$. Tuberc., 42, 58.

MARRIS, E., et al. (1952), Ұ.A.M.A.; I49, 1120. Stratton, New York.

RILANCE, A. B., and WARRING, F. C. (1944), Am. Rev. Tub., SCADDDING, F. H., NICHOLSON, H., and HOYLE, C. (195I), Quart. F. Med., N.S. 2, 313 .

THOMAS, D. M. E. (1950), Personal communication.

WRIGHT, G. W., et al. (1949), Ұ. Thor. Surg., 18, 372.

VAJDA, L. (1933), Ztschr. f. Tuberk., 67, $37 \mathrm{I}$. 